\title{
Daily negative interactions and mood among patients and partners dealing with multiple sclerosis (MS): The moderating effects of emotional support
}

\author{
Annet M. Kleiboer ${ }^{\mathrm{a}, *}$, Roeline G. Kuijer ${ }^{\mathrm{b}}$, Joop J. Hox ${ }^{\mathrm{c}}$, Peter J.H. Jongen ${ }^{\mathrm{d}}$, \\ Stephan T.F.M. Frequin ${ }^{\mathrm{e}}$, Jozien M. Bensing ${ }^{\mathrm{f}}$ \\ ${ }^{a}$ Utrecht University, Faculty of Social Sciences, Clinical and Health Psychology, P.O. Box 80140, 3508 TC Utrecht, The Netherlands \\ ${ }^{\mathrm{b}}$ University of Canterbury, Christchurch, New Zealand \\ ${ }^{\mathrm{c}}$ Utrecht University, Utrecht, The Netherlands \\ ${ }^{\mathrm{d}}$ MS Centre Nijmegen, Nijmegen, The Netherlands \\ ${ }^{\mathrm{e}}$ St. Antonius Hospital, Nieuwegein, The Netherlands \\ ${ }^{\mathrm{f}}$ Nivel, Utrecht, The Netherlands
}

Available online 29 September 2006

\begin{abstract}
Negative interactions with intimate partners may have adverse consequences for well-being, especially for individuals dealing with chronic illness. However, it is not clear whether negative interactions affect both dimensions of positive and negative well-being and factors that may moderate this effect have not been well-described. The aim of the present study was to examine the association between daily received negative responses from the partner and end-of-day positive and negative mood in patients with multiple sclerosis (MS) and their intimate partners. Further, the moderating role of receiving emotional support from the partner on the same day was examined. Sixty-one MS patients and their intimate partners were approached via one MS centre and the neurology department of one hospital in the Netherlands and completed computerized diaries for 14 days. Both partners filled out diaries at the end of each day, recording received negative responses, emotional support and end-of-day positive and negative mood. In line with a domain specific model, patients or partners who reported receiving negative responses on a day had higher end-of-day negative mood, whereas received negative responses were unrelated to end-of-day positive mood. Further, for both patients and partners, the adverse effect of received negative responses on end-of day mood was moderated by receiving emotional support on the same day.
\end{abstract}

(C) 2006 Elsevier Ltd. All rights reserved.

Keywords: Negative interactions; Emotional support; Multiple sclerosis; Diary method; Couples; Mood

\section{Main text}

The beneficial effects of being involved in a supportive relationship for well-being in general

\footnotetext{
*Corresponding author.

E-mail address: a.m.kleiboer@fss.uu.nl (A.M. Kleiboer).
}

(e.g. Burman \& Margolin, 1992) as well as for adjustment to chronic illness (e.g. Cutrona, 1996) have been well documented. Unfortunately, besides being supportive, interactions with the partner can also be problematic and the detrimental effects of problematic or negative interactions are believed to be more important than the beneficial effects of 
supportive interactions (Manne, Taylor, Dougherty, \& Kemeny, 1997; Newsom, Nishishiba, Morgan, \& Rook, 2003; Schuster, Kessler, \& Aseltine, 1990). The aim of the present study was to examine the association between negative responses received from the partner and end-of-day mood in patients with multiple sclerosis (MS) and their intimate partners. Additionally it was examined whether this relation was moderated by receiving emotional support from the partner that same day.

By and large, the literature examining the impact of negative interactions in the context of chronic illness has focused on the patient. Negative partner interactions may involve well-intended support attempts, such as minimizing or maximizing the consequences of the illness (Lehman \& Hemphill, 1990) or being overprotective (Cutrona, 1996; Kuijer et al., 2000), but they may also include more overtly negative interactions, such as being critical, avoidant or demanding (Rook, 1992; Rook \& Pietromonaco, 1987). Although these overtly negative responses are not common, they have been reported by patients with various chronic illnesses, such as cancer (Manne et al., 1997), rheumatoid arthritis (Revenson, Schiaffino, Majerovitz, \& Gibofsky, 1991) and MS (Lehman \& Hemphill, 1990), with adverse effects on well-being. Negative responses from the patient towards the healthy partner and the effects on the healthy partner's wellbeing have largely been neglected, however. The present study focused on the more overt negative responses that both partners may display towards each other.

One explanation for the strong impact of negative interactions on well-being is that they may be a source of stress themselves (Shinn, Lehmann, \& Wong, 1984). In a diary study examining several daily stressors, Bolger, DeLongis, Kessler, and Schilling (1989) found that daily interpersonal stressors (i.e. conflicts, demands) were the most distressing events. Negative interactions may be interpreted as a sign of rejection by the recipient and are believed to have such great impact because they entail a threat both to the relationship with the provider and to psychological resources such as selfesteem (Rook, 1992). Individuals whose psychological resources to deal with stressful events are already depleted, such as in individuals dealing with chronic illness and their intimate partners, may be particularly vulnerable to negative responses they receive from the partner (Druley \& Townsend, 1998; Ingersoll-Dayton, Morgan \& Antonucci, 1997).
MS is a chronic and often disabling disease of the central nervous system, characterized by an unpredictable and variable course. MS is most commonly diagnosed in young adulthood. The onset can be gradual or acute and in most patients relapses are followed by periods of remission. However, a progressive decline in health is possible as well (Lublin \& Reingold, 1996). Due to the nature of MS, patients constantly have to adapt to new situations, often involving losses such as reduced physical independence, and restrictions in daily activities and social relationships. Compared to patients with other illnesses, higher levels of depression and lower levels of self-esteem have been documented in MS patients (Murray, 1995).

The severity of the disease, the unpredictability of its course and the lack of specific treatment not only present a considerable challenge to the patient, but also to the intimate partner (Pakenham, 1998). Because of their interdependence, both members of the couple are affected not only by their own reactions to the illness but also by the emotional distress of their partner (Kelley \& Thibaut, 1978; cf. Rusbult \& Van Lange, 2003). Research has shown that the well-being of patients and partners is highly related and often follows the same pattern over time (Pakenham, 1998; Segrin et al., 2005). Additionally, for partners, taking care of an ill partner often implies taking on additional responsibilities such as performing household tasks or assistance with selfcare. Ample research has shown that taking care of an ill partner may be burdensome (e.g. Pakenham, 1998; Ybema, Kuijer, Hagedoorn, \& Buunk, 2002).

Thus, both patients and their intimate partners may have depleted resources to deal with stressful events and may therefore be psychologically vulnerable to negative responses they receive from the partner. Support for this notion was found in a study by Druley and Townsend (1998). Negative responses received from the partner were related to depression through self-esteem in rheumatoid arthritis patients, but not in healthy controls. Additionally, a study by Kiecolt-Glaser, Dyer, and Shuttleworth (1988) showed that upsetting interactions with others were related to higher levels of depression in caregivers of Alzheimer patients, but not in healthy controls. Although this study was not limited to partner caregivers and did not address negative responses from the partner specifically, it suggests that healthy partners caring for an ill partner may have increased vulnerability for negative responses as well. 
With respect to effects on well-being, different association patterns have been suggested between negative responses received from others and positive and negative dimensions of well-being. Some researchers suggest a domain specific effect, that is, negative responses impact on dimensions of negative well-being but not on dimensions of positive well-being (e.g. Ingersoll-Dayton et al., 1997). However, other researchers argue that the effects of negative responses are so potent that they affect both positive and negative well-being, which is termed a cross-over effect (e.g. Finch, Okun, Barrera, Zautra, \& Reich, 1989). Empirical support for both models has been found (Finch et al., 1989; Finch, Okun, Pool, \& Ruehlman, 1999; IngersollDayton et al., 1997; Manne et al., 1997; Rook, 2001). If a cross-over effect is engendered by the potency of negative responses, as some researchers suggest, one might expect that this model applies especially to individuals who are vulnerable to negative responses, that is, those who are dealing with chronic illness and their intimate partners.

However, although both patients and partners may be vulnerable to negative interactions with the partner, this may be more pronounced among patients. When one partner is diagnosed with chronic illness, the relationship is likely to change in such a way that the ill partner becomes more reliant on the healthy partner, not only for practical assistance but also with respect to their self-esteem (Cutrona, 1996; Druley \& Townsend, 1998). For patients, other sources of esteem (such as work) are often lost and the partner may compensate for these losses by communicating that the patient is still valued and cared for. Because of this higher dependency on the partner, patients may be particularly vulnerable to negative responses received from their partner (Rusbult \& Van Lange, 2003).

Whereas most studies so far used global selfreport questionnaires to examine the impact of negative responses on well-being, this study used a diary method as we were specifically interested in the impact of negative interactions on daily mood. A diary method is an appropriate instrument to address the emotional reaction elicited by negative responses received from the partner during the course of daily life (Bolger, Davis, \& Rafaeli, 2003). Mood was taken as an outcome measure because it has been found to be sensitive to distress provoked by daily occurrences such as support interactions (Stone, 1997; Stone, Neale, \& Shiffman, 1993). The distress that is experienced as a consequence of negative interactions is believed to aggregate to influence broader dimensions of health (Rook, 2001). Moreover, for MS patients, investigating negative interactions is particularly important as it is suggested that stress (e.g. negative interactions) is related to periods of disease activity in MS (Schwartz et al., 1999).

A diary method permits a description of day-today fluctuations in mood and compares end-of-day mood on days that individuals did or did not report receiving negative responses. With this method a fine-grained analysis of the effects of negative interactions is possible. Additionally, a diary method has some methodological advantages as compared to single-point self-report questionnaires. First, the problem of retrospection is minimized by reducing the time between actual occurrence and report (West \& Hepworth, 1991). Second, by examining the temporal precedence of events within individuals, participants can be used as their own controls, thereby reducing the influence of confounding variables (West \& Hepworth, 1991).

Considering the profound effects of negative responses on well-being, it is important to explore conditions that moderate this effect. The present study examined whether the effects of negative responses received from the partner were reduced when participants received emotional support from their partner on the same day. Receiving negative responses from the partner does not preclude that one receives emotional support from the partner (Revenson et al., 1991; Vinokur \& Van Ryn, 1993). Emotional support involves the expression of love and care by communicating affection, value and interest (Helgeson \& Cohen, 1996). As was mentioned previously, receiving negative responses from the partner may be viewed as a sign of rejection by the recipient. It is reasonable to assume that the impact of negative responses will be less threatening to the relationship and to psychological resources (i.e. participants feel less rejected) when it is given by a partner who communicates love and care on the same day.

Thus far, most studies that considered the joint effects of positive and negative support in the partner relationship did not find a buffering effect of emotional support on the association between received negative responses and well-being (Manne et al., 1997; Schuster et al., 1990). However, these studies were typically based on between-person designs, that is, they examined whether individuals 
who in general receive high levels of negative responses from their partner are less affected by these negative responses if the partner in general provides much positive support as well (here, we use the term emotional support to refer to positive support and the term negative response to refer to negative support). Such a between-person design is different from the within-person design that was used in the current study. A within-person design provides information on the effects of negative responses when they occur or about conditions under which they have a larger effect (e.g. when emotional support is provided as well on the same day). For example, it may be that patients and partners do not differ with respect to the amount of negative responses they receive in general, but that patients are more strongly affected by them when they occur. Additionally, it may be that the effects of receiving negative responses are dependent on other things that happen on the same day (i.e. receiving emotional support).

In sum, this study examined the impact of negative responses received from the partner on end-of-day positive and negative mood in MS patients and their intimate partners. Further, the moderating role of receiving emotional support on the same day was examined. Both for patients and partners, we expected that negative responses received from the partner would have a negative effect on both positive and negative dimensions of mood, however, we expected that the effects were more pronounced for patients. Additionally, both for patients and partners, we expected that the effects of received negative responses would be moderated by receiving emotional support as well on the same day.

\section{Method}

\section{Participants}

MS patients and their intimate partners were approached via one MS centre and the neurology department of one hospital in the Netherlands. MS patients who were registered in the patient files of these clinics received a letter including information about the study and a description of the inclusion criteria. As information on inclusion criteria were not available from the patient files (e.g. we did not know how many of these patients had a partner), we distributed letters to all 390 patients that were registered. This means that the number of couples who responded to the information letter could not be used to calculate response rates. Couples were eligible for inclusion if: (a) one partner was diagnosed with MS; (b) patients were currently involved in a heterosexual relationship with minimum relationship duration of one year; (c) patients were living together with their intimate partner, and (d) both partners were willing to participate in the study. Exclusion criteria were insufficient understanding of the Dutch language or when one or both partners suffered from serious psychiatric or medical problems other than MS.

Seventy couples, who were eligible for inclusion, responded to the information letter and signed informed consent. Of the 70 couples, a final sample of 61 couples completed all the materials. Reasons mentioned for drop-out were MS related health problems $(n=3)$ and personal reasons $(n=3)$. Three couples dropped-out without additional comments. Descriptive characteristics of the sample are detailed in Table 1 (second and fifth column). The sample showed wide variance in age, year since diagnosis and relationship length. Additionally, more female patients participated in the study which reflects the distribution of gender in the population. Further, $31 \%$ of the patients were employed (12\% full-time and $20 \%$ part-time); the other patients received a disability benefit $(51 \%)$, performed household duties $(10 \%)$ or were unemployed for other reasons $(8 \%)$. Additionally, most partners $(80 \%)$ were employed $(61 \%$ full-time and $19 \%$ part-time), the other partners were retired $(11 \%)$ or were unemployed for other reasons $(9 \%)$. Twenty-three percent of the patients and $27 \%$ of the partners had completed the lowest level of secondary (vocational) education only, $41 \%$ of the patients and $40 \%$ of the partners had completed middle to higher levels of secondary (vocational) education, and $36 \%$ of the patients and $33 \%$ of the partners had a college degree or higher.

Patients reported their actual disability by means of a self-report checklist (Kurtzke, 1981). Problems mentioned by patients included, amongst others, fatigue $(95 \%)$, limited walking ability (56\%), bladder problems $(72 \%)$, bowel problems $(42 \%)$, visual problems $(25 \%)$ and speech problems $(15 \%)$. In general, the sample varied widely considering their physical disability, some patients were only mildly affected whereas others were severely impaired (mean 14, range 1-40, on a 0-64 scale, with higher scores indicating more disability). Additionally, $57 \%$ of the patients described their MS as 
Table 1

Means (SD) of patients and partners who did or did not report receiving negative responses from their partner across the 14-day period

\begin{tabular}{|c|c|c|c|c|c|c|}
\hline & \multicolumn{3}{|l|}{ Patients } & \multicolumn{3}{|l|}{ Partners } \\
\hline & $\begin{array}{l}\text { Total } \\
N=61\end{array}$ & $\begin{array}{l}\mathrm{No}^{\mathrm{a}} \\
N=18\end{array}$ & $\begin{array}{l}\text { Yes }^{\mathrm{b}} \\
N=43\end{array}$ & $\begin{array}{l}\text { Total } \\
N=59\end{array}$ & $\begin{array}{l}\mathrm{No}^{\mathrm{a}} \\
N=13\end{array}$ & $\begin{array}{l}\mathrm{Yes}^{\mathrm{b}} \\
N=46\end{array}$ \\
\hline Gender, \% women & $87 \%$ & $89 \%$ & $86 \%$ & $14 \%$ & $15 \%$ & $8 \%$ \\
\hline Age & $46.8(8.5)$ & $50.8(5.9)$ & $45.0(8.9)^{* *}$ & $49.3(9.3)$ & $51.2(11.7)$ & $48.8(8.6)$ \\
\hline Relationship duration & $22.6(10.0)$ & $23.8(9.5)$ & $22.1(10.3)$ & $22.8(10.3)$ & $21.0(11.8)$ & $23.2(10.0)$ \\
\hline Education (1-8 scale) & $4.5(2.3)$ & $3.5(2.1)$ & $4.9(2.2)^{*}$ & $4.6(2.2)$ & $4.3(2.2)$ & $4.7(2.3)$ \\
\hline Year since diagnosis & $8.3(6.0)$ & $7.6(4.7)$ & $8.5(6.5)$ & & & \\
\hline Physical disability (0-64 scale) & $14.2(9.5)$ & $13.2(8.3)$ & $14.6(10.0)$ & & & \\
\hline Daily MS related symptoms ( $1-10$ scale) & $4.8(2.2)$ & $5.0(2.4)$ & $4.8(2.1)$ & & & \\
\hline Daily Hassles (0-22 scale) & $4.4(2.5)$ & $2.9(2.1)$ & $5.1(2.3)^{* *}$ & $4.1(3.4)$ & $2.4(2.8)$ & $4.6(3.5)^{*}$ \\
\hline Daily positive mood ( $10-50$ scale) & $24.8(5.8)$ & $23.5(6.1)$ & $25.3(5.6)$ & $25.4(5.5)$ & $25.2(6.2)$ & $25.4(5.4)$ \\
\hline Daily negative mood (10-50 scale) & $14.5(4.0)$ & $14.3(5.2)$ & $14.6(3.5)$ & $14.1(4.1)$ & $13.2(2.3)$ & $14.3(4.5)$ \\
\hline Emotional support $(0-3$ scale $)$ & $1.8(.8)$ & $1.5(.9)$ & $2.0(.63)$ & $1.5(.8)$ & $1.8(1.0)$ & $1.5(.72)$ \\
\hline
\end{tabular}

Note: $* p<.05, * * p<.01 T$-tests were used to compare those who did and did not report receiving negative responses.

${ }^{\text {a }}$ No: participants who did not report receiving negative responses across the 14-day period.

${ }^{\mathrm{b}}$ Yes: participants who reported receiving negative responses over the 14-day period.

having a relapse remitting course, $43 \%$ as having a progressive course.

\section{Procedure}

Prior to the diary part of the study, patients and partners completed questionnaires concerning demographics and disease related characteristics at home. Next, the couples were visited by the first author or a research assistant to provide additional information and instructions about the diary part of the study and to install an electronic diary on the couple's computer. Participants who did not own a computer were provided with a computer from the university. Computer software was developed especially for this study. The electronic diary was userfriendly and easy to complete. Even participants with little or no experience with computers were able to use the program after they were given clear instructions. Participants were instructed to complete the electronic diary every evening before going to bed, starting the following day. They were asked to complete the diary for 14 days. Participants were explicitly asked not to share the answers with their partner while completing the diaries. The diary was designed to be completed in 5-10 min. Recordings were saved on a floppy disk and the participants did not have access to their reports after they were saved. After two weeks, the couples returned the floppy disk containing each night's responses by mail.

To verify compliance, the date and time of recordings were saved on the floppy disk. Partici- pants were allowed to complete the diary the next morning if they did not manage to do it at night $(2.6 \%$ of the diaries were completed the next morning). Diaries that were completed too early (before $2 \mathrm{pm}$ ) or too late (after $2 \mathrm{pm}$ the next day) were considered unreliable and were excluded from further analysis (1.6\% of the recordings). Further, participants who completed less then 10 days during the 14 day period were excluded from further analyses. For this reason, two healthy partners were excluded who completed 6 and 9 days of recordings, respectively. An average of 13.1 days of recordings was reported across the 14 day period.

\section{Instruments}

Negative responses were assessed each evening. Both patients and partners reported if and to what extent they had received negative responses from their partner that day. Negative responses were assessed with four items including the following questions: Did it happen today that your partner: 'made disapproving remarks of you?', 'avoided you?', 'hurt your feelings?', and 'demanded a lot of you?'. All answers were given on a four-point scale $(0=$ not at all, $3=$ very much). The four items were averaged to form a negative received support scale. However, the occurrence of negative responses was rare and participants almost never used the higher scores of the scale which resulted in strongly skewed distributions. Therefore, the scale was dichotomized into one variable that indicated 
the absence or presence of a negative response on a certain day. Consequently, we did not distinguish between the effects of the different items, nor did we differentiate between the strength of the responses that were reported. However, we considered this justified as the correlations between the four items and the dependent variables positive and negative mood were similar in magnitude and direction ${ }^{1}$.

Emotional support was assessed each evening as well. Both patients and partners reported if and to what extent they had received emotional support from their partner that day. Three items were used to measure emotional support including the following questions: Did it happen today that your partner: 'listened to you?', 'was affectionate towards you?', 'gave you a compliment?'. All answers were given on a four-point scale $(0=$ not at all, $3=$ very much). Cronbach's alpha based on the aggregated scores was .84 for patients and .85 for partners.

End-of-day mood was measured with the positive and negative affect schedule (PANAS; Watson, Clark, \& Tellegen, 1988). The instrument consists of 10 positive and 10 negative adjectives that make up a negative and positive mood scale. Each evening, participants were asked to indicate to what degree they experienced the respective emotion 'right now', on a five-point scale ranging from (1) slightly or not at all to (5) extremely. Scores on the positive items and the negative items were summed per day. Based on the aggregated scores, Cronbach's alpha was .87 and .83 for the positive scale in patients and partners, respectively, and .81 and .76 for the negative scale in patients and partners, respectively. The correlation between positive and negative mood over days within patients and partners was close to zero $(r=.12$ and $r=.05$, respectively).

The number of daily hassles experienced each day was included as a control variable since hassles may influence the receipt of negative responses and emotional support. A checklist was used based on the Revised Hassle and Uplift Scale (DeLongis, Folkman, \& Lazarus, 1988). However, we considered the original 53 item scale too long to be used on a daily basis, so we shortened the scale by conjoining several items. One item referring to the

\footnotetext{
${ }^{1}$ Correlations over persons and days between positive mood and the four items, 'hurt feelings', 'criticism', 'demanding' and 'avoidance' were $-.01, .03, .01$, and .01 in patients and -.01 , $-.02, .06$ and .06 in partners, respectively. Correlations between negative mood and the four items 'hurt feelings', 'criticism', 'demanding' and 'avoidance' in patients were $.28, .21, .18$, and .16 and in partners were $.39, .32, .21$, and .26 respectively $(p<.01)$.
}

occurrence of special incidents (e.g. accident, winning the lottery) was added. This resulted in a 22 item scale that represented all the areas that were included in the original scale.

Finally, the amount of MS related symptoms that a patient experienced was included as a control variable for the same reason as daily hassles. Patients were asked each evening to indicate to what extent they had experienced MS related symptoms that day. The answer was given on a ten-point scale ranging from (1) no symptoms to (10) many symptoms.

\section{Analyses}

Multi-level regression modelling, as implemented in the program MLwiN (Rasbash, Browne, Goldstein, \& Yang, 2000) was used to determine the relationship between the predictors and dependent variables. Each model had three levels, a between-couple level (level 3), a between-person level (level 2) and a withinperson level (level 1). The within-person level of the analyses allowed each individual's evening mood on a given day to be modelled as a function of that individual's received negative responses ${ }^{2}$. We predicted end-of-day mood while controlling for previous day end-of-day mood, daily hassles, patient's MS related symptoms and received emotional support.

The analyses were started with an intercept only model that separated the variance of end-of-day mood in three independent components: mood-level variance, person-level variance and couple-level variance. In this study we were primarily interested in the predictive value of negative responses on endof-day mood. Therefore we reported the amount of variance explained by the predictors at the daily level only. It is important to note that variance explained at the lowest level (that is days) cannot be explained by variables at higher levels, because these are constant at the days-within-persons level. Next, the predictors were entered in the regression equation hierarchically, starting with the control variables (step 1), followed by role (step 2), the main effects of emotional support and negative responses (step 3), the interaction between emotional support and negative responses (step 4), and the two-way and three-way interactions between role, emotional

\footnotetext{
${ }^{2}$ In additional analyses we investigated the lagged effects of negative interactions on mood on the following days. However, we did not find evidence for an effect beyond same day end-ofday mood.
} 
support and negative responses (step 5). All predictors were grand mean centred (i.e. the overall mean was subtracted from the values of a variable), thus the regression intercept represented mood when all variables were at their average level. The variables negative responses received from the partner and role (patient or partner) were effect coded. No negative responses received on a day was coded as -.5 and negative responses received as .5 . Additionally, patients were coded as -.5 and partners as .5. When interaction effects were significant, regression equations were estimated for days on which individuals were scoring $1 S D$ below and $1 S D$ above the mean of the interaction variables (Aiken \& West, 1991), to obtain a graphical diagram of the interaction.

\section{Results}

\section{Descriptive analyses}

Table 1 presents the means and standard deviations of the key study variables. Across the 14-day period, 18 patients $(30 \%)$ and 13 partners $(21 \%)$ did not report receiving negative responses from their partner. The mean number of days that patients or partners reported receiving negative responses was 2.6 (range 0-11) and 3.8 (range 0-13), respectively. Thus, across the 14-day period, partners reported more days on which they received negative responses from their ill partner than patients $(p<.05)$. Additionally, all participants reported receiving emotional support from their partner across the 14-day period.
As shown in Table 1, patients who did not report receiving negative responses across the 14-day period were older, had lower education and experienced less daily hassles than patients who did report receiving negative responses across the 14-day period. Further, partners who did report receiving negative responses across the 14-day period differed from partners who did not report receiving negative responses on the amount of daily hassles they reported. In line with the results for patients, they reported experiencing more daily hassles. Apparently, individuals who reported receiving negative responses from their partner reported more daily hassles in general. Individuals who did or did not report receiving negative responses across the 14-day period did not differ from each other on other key study variables such as the amount of positive and negative mood, or the amount of emotional support that they reported receiving.

Based on the daily measures, the correlation between received negative responses and emotional support as reported by MS patients and their partners was -.01 and -.02 , respectively. This suggests that patients or partners who reported receiving high levels of emotional support, did not necessarily also report receiving low levels of negative responses on the same day.

\section{End-of-day positive mood}

Table 2 presents the results of the multilevel analysis predicting end-of-day positive mood as a function of received negative responses and emotional support. The intercept indicates that the predicted amount of end-of-day positive mood for

Table 2

The effects of received negative responses from the partner on end-of-day positive and negative mood in patients and the moderating role of received emotional support

\begin{tabular}{|c|c|c|c|c|c|c|}
\hline & \multicolumn{3}{|c|}{ Positive mood } & \multicolumn{3}{|c|}{ Negative mood } \\
\hline & $B$ & SE & Beta & $B$ & SE & Beta \\
\hline Intercept & 24.92 & .53 & & 14.65 & .28 & \\
\hline Step 1 Previous day mood & .16 & .03 & $.16^{* * *}$ & .21 & .02 & $.21 * * *$ \\
\hline MS related symptoms & -.53 & .10 & $-.18^{* * *}$ & .51 & .07 & $.24 * * *$ \\
\hline Hassles & -.08 & .07 & -.04 & .40 & .05 & $.26^{* * *}$ \\
\hline Step 2 Role (patient/partner) & .81 & .69 & .05 & -.50 & .49 & -.05 \\
\hline Step 3 Emotional support & 1.43 & .27 & $.17 * * *$ & -.27 & .18 & -.05 \\
\hline Negative responses & -.13 & .43 & -.01 & 1.58 & .29 & $.12 * * *$ \\
\hline Step 4 Emo*neg & .40 & .44 & .02 & -.85 & .29 & $.07 * * *$ \\
\hline Step 5 Role*emo & ns & & & ns & & \\
\hline Role*neg & ns & & & ns & & \\
\hline Role*emo*neg & ns & & & ns & & \\
\hline
\end{tabular}

Note: $* * * p<.001$. Interactions between role and the main effects are presented only when significant. 
an average person on an average day was 25 (on a $10-50$ scale). Additionally, the variance estimates of the intercept-only model showed that $51 \%$ of all variance in the data set was explained at the daily level. In the first step, the control variables were entered. Better previous day end-of-day positive mood and less MS related symptoms in patients were related to better end-of-day positive mood whereas the amount of daily hassles experienced was unrelated to positive end-of-day mood. Together the control variables accounted for $7 \%$ of the variance at the daily level. In the second step, role (patient or partner) did not contribute significantly to end-of-day positive mood, indicating that patients and partners did not differ with respect to their positive end-of-day mood. In the third step, the main effects of emotional support and negative support were entered. Individuals reported better end-of-day mood when they received more emotional support, whereas end-of-day positive mood was unrelated to received negative responses. Together, received emotional support and negative responses accounted for $2 \%$ of the variance in end-of-day positive mood. In the fourth step, the interaction between emotional support and negative responses was entered to examine whether the effects of negative responses differed as a function of emotional support. The interaction variable did not contribute significantly to the model (the explained variance was $0 \%$ ). Finally, the interactions between negative responses and emotional support on the one hand and role on the other hand were entered in order to examine whether the effects of negative responses and emotional support and the interaction term differed as a function of role. However, none of the effects were dependent on role, that is, the effects of emotional support and negative responses were the same for patients and partners.

In sum, both for patients and partners, received emotional support was related to end-of-day positive mood whereas received negative responses was unrelated to end-of-day positive mood.

\section{End-of-day negative mood}

Table 2 presents the final model of the multilevel analysis predicting end-of-day negative mood as a function of received negative responses and emotional support. The intercept indicates that the predicted amount of end-of-day negative mood for the average person on an average day was 15 (on a 10-50 scale). The intercept-only model showed that

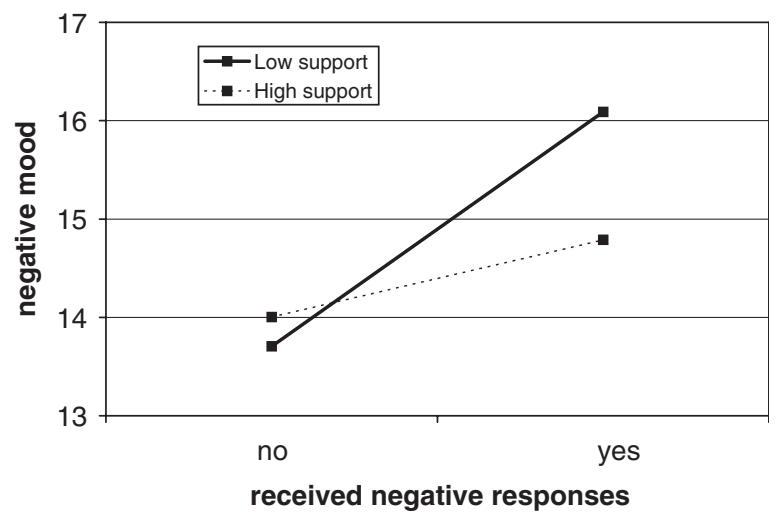

Fig. 1. The relation between received negative responses and endof-day negative mood moderated by received emotional support in both patients and partners.

$50 \%$ of all variance in the data set was explained at the daily level. In the first step, the control variables were entered and contributed all significantly to the equation. Worse previous day end-of-day mood, more MS related symptoms and more hassles were related to worse end-of-day negative mood which accounted for $12 \%$ of the variance in end-of-day negative mood. In the second step, role (patient or partner) did not contribute significantly to end-of-day negative mood, that is, patients and partners did not differ with respect to their end-of-day negative mood. Next, the main effects of emotional support and negative support were entered. Individuals reported worse end-of-day negative mood when they received negative responses from their partner, but received emotional support was not related to end-of-day negative mood. The explained variance at the daily level was $5 \%$. In the fourth step, the interaction between emotional support and negative responses was entered. The interaction contributed significantly to the regression and explained $1 \%$ of the variance at the daily level. As is shown in Fig. 1, the effect of negative responses received from the partner on endof-day negative mood was reduced when patients and partners reported receiving high levels of emotional support on the same day as well $(b=2.38, p<.00$ when low levels of emotional support were received; $b=.78, p=.05$ when high levels of emotional support were received). Finally, the interactions between negative responses and emotional support on the one hand and role on the other hand were entered. Again, no significant differences between patients and partners were found.

In sum, both for patients and partners, the effect of negative responses received from the partner was 
reduced when patients or partners received emotional support from the partner as well.

\section{Discussion}

The present study examined daily received negative responses in MS patients and their intimate partners. First, the relation between daily received negative responses from the partner and end-of-day mood was examined. Second, the moderating role of receiving emotional support on the same day was addressed. An important feature of the study was the use of a diary method which allowed us to investigate the effects of negative responses withinpersons over time.

In line with other studies, both patients and partners reported receiving few negative responses from their partner and some patients and partners did not report receiving any negative responses across the 14-day period (e.g. Rook, 2001). In addition, it was found that patients and partners who reported receiving negative responses from the partner reported more daily stress in general, which is in line with results documented by Rook (2003). She reported that greater exposure to negative responses from others was related to higher life stress in older adults. Apparently, individuals who report receiving negative responses from their partner report more daily hassles in general. Further, patients who reported receiving negative responses from the partner were younger and higher educated. A comparable relation between age and exposure to negative interactions than partners was found in a study by Krause and Shaw (2002).

In concordance with a domain specific model, the impact of received negative responses from the partner was limited to negative mood in both patients and partners. Received negative responses from the partner detracted from end-of-day negative mood but were unrelated to end-of-day positive mood. We expected a cross-over effect of received negative responses on mood in both patients and partners. This assumption was based on the potent effects of negative responses and the higher vulnerability to these responses in individuals under stress, such as in patients and partners dealing with MS. One reason that may explain why we did not find a cross-over effect of received negative responses may be that the effects of negative responses on mood were relatively weak as compared to the effects of MS related symptoms and daily hassles, for example. It may be that cross-over effects are only found when effects are potent. The received negative responses variable was dichotomized because of the low frequency in our sample. As a result we were not able to distinguish between days that participants reported receiving many negative responses and days that participants reported receiving few negative responses, which may have decreased the power of our predictor. However, despite a decrease in power, we found an important effect of received negative responses on end-of-day negative mood over and above daily hassles and MS related symptoms.

We did not find evidence that the effects of received negative responses were different for patients and partners. We expected that patients would be more vulnerable to negative interactions than partners because they are often placed in a position of dependence and more dependent on their partner for support (Rusbult \& Van Lange, 2003). As far as we know, this is the first diary study that compared patient's and partner's vulnerability to received negative responses from the partner. Several studies have shown that patient's and partner's well-being is highly related and follows the same pattern during the course of illness (Pakenham, 1998; Segrin et al., 2005). Our results suggest that the effects of received negative responses from the partner are similar for both patients and their intimate partners.

The present study focused on the effects of negative interactions on same day end-of day mood. However, it is possible that these negative interactions have an effect on mood beyond the day on which they occur. We performed additional analyses to examine this issue (see note 2), but found no support for such an effect. These findings are in line with studies examining the association between daily stress and mood, which generally do not find an effect of stress on mood beyond the day as well (Stone, Neale, \& Shiffman, 1993). This is interesting considering the apparent importance of negative responses for long term well-being (e.g. Rook, 2001). As suggested by Stone and colleagues (1993), it may be that the impact of the events that were reported was not large enough or that an effect beyond the day only exists in some individuals, such as in individuals with high interpersonal sensitivity (Smith \& Zautra, 2002). Additionally, there may be complex patterns of associations between received negative responses and mood that are cumulative and exceed over days or months. One aspect that may be important in this context is the frequency of days that individuals report receiving negative 
responses. Our sample showed a large variance in the frequency of days that patients and partners reported receiving negative responses from the partner. An interesting challenge for future research is to examine how daily negative responses affect long term well-being.

Our results showed that the impact of received negative responses from the partner on end-of-day negative mood can be buffered by receiving high levels of emotional support on the same day, both for patients as well as for partners. Our data does not reveal whether the emotional support is provided concurrently with, before or after the negative responses. However, it was shown that the adverse effects of received negative responses from the partner were reduced when individuals received high levels of emotional support as well within a relatively short time period (one day). The present study does not allow us to examine why emotional support buffers the effect of received negative responses. For example patients or partners may feel less rejected when they receive emotional support in addition to negative responses. Additionally, receiving negative responses may be less threatening to the relationship when emotional support is provided as well because, despite the negative responses, the partner knows that he or she is still liked by the other partner. Further, the present study showed that emotional support is important, not only directly but also in buffering the effects of receiving negative responses from the partner. Interventions should teach patients and partners about these mechanisms. They should not only focus on reducing negative interactions but they should also attend to mechanisms that can prevent negative responses from having its harmful effects.

Some limitations of the present study require attention. First, a diary method offers the unique opportunity to examine the effects of support processes across time. However, the non-experimental nature of the data precludes causal statements. As recordings of support and mood were collected at the same time (i.e. end-of-day), it is possible that patients or partners who were in a better mood were also inclined to report receiving more or less negative responses or emotional support. However, we controlled for important confounding variables, that is, previous day endof-day mood, daily hassles and MS related symptoms. Herewith, we protected to some extent against the problem of mood simply predicting support interactions. Further, the selection of the 14-day period for the diary measures was inherently arbitrary and we cannot be sure that the 2 week period we selected was characteristic for the couple's daily life. This is an intrinsic problem to diary studies. However, to protect against this problem the couples were instructed to complete the diaries during a period that was representative for their daily life with respect to, for example, days at work.

Further, given that the majority of our sample consisted of female patients and male partners, we were unable to address gender differences. Some of our findings may have been different if we had considered male patients and female partners. Additionally, we do not know whether the pattern of findings is specific to couples facing MS or whether the findings can be generalized to other chronic diseases. We assume that our findings apply to other resembling diseases as well, such as rheumatoid arthritis. Further, the MS patients in our study did not experience relapses during the 14-day period. The effects of negative responses received from the partner may be different during crisis (i.e. flare ups). Other limitations concern the small sample size and the fact that we do not know whether our sample represented couples dealing with MS. It is possible that distressed couples were less willing to participate in a study like this. Related to this, on average the couples in this sample had been together for a long time suggesting that they somehow managed to deal with the MS in their relationship successfully.

Finally, the effects of negative responses and the moderating role of emotional support may depend on the individual. Indeed, our data suggested that the effects of received negative responses and emotional support varied as a function of between-person differences. However, we did not examine this any further because it was beyond the scope of this study. Variance explained by between-person differences means that certain aspects of the patient or partner moderates the effects of received negative responses or emotional support. For example, previous studies have suggested that reactivity to negative responses were dependent on an individual's self-esteem (Rook, 2003) or interpersonal sensitivity (Smith \& Zautra, 2002).

To conclude, the present study adds to a growing body of research that describes the detrimental effects of receiving negative responses from the 
partner on end-of-day mood. Interestingly, our results indicated that the consequences of receiving negative responses from the partner were for a large part dependent on the amount of emotional support that patients or partners received from their partner on the same day. Further, the study showed that examining daily within-person processes over time may provide us with valuable information considering the conditions under which the adverse impact of negative interactions may be more or less profound.

\section{Acknowledgments}

The authors thank Martine van Tent for her help with the data collection and Jan Koopman for developing the software for the electronic diaries.

\section{References}

Aiken, L. S., \& West, S. G. (1991). Multiple regression: Testing and interpreting interactions. Newbury Park, CA: Sage.

Bolger, N., Davis, A., \& Rafaeli, E. (2003). Diary methods: Capturing life as it is lived. Annual Review of Psychology, 54, 579-616.

Bolger, N., DeLongis, A., Kessler, R. C., \& Schilling, E. A. (1989). Effects of daily stress on negative mood. Journal of Personality and Social Psychology, 57(5), 808-818.

Burman, B., \& Margolin, G. (1992). Analysis of the association between marital relationships and health problems: An interactional perspective. Psychological Bulletin, 112(1), $39-63$.

Cutrona, C. E. (1996). Social support in couples: Marriage as a resource in times of stress. Thousand Oaks, CA: Sage.

DeLongis, A., Folkman, S., \& Lazarus, R. S. (1988). The impact of daily stress on health and mood: Psychological and social resources as mediators. Journal of Personality and Social Psychology, 54(3), 486-495.

Druley, J. A., \& Townsend, A. L. (1998). Self-esteem as a mediator between spousal support and depressive symptoms: A comparison of healthy individuals and individuals coping with arthritis. Health Psychology, 17(3), 255-261.

Finch, J. F., Okun, M. A., Barrera, M., Zautra, A. J., \& Reich, J. W. (1989). Positive and negative social ties among older adults: Measurement models and the prediction of psychological distress and well-being. American Journal of Community Psychology, 17(5), 585-605.

Finch, J. F., Okun, M. A., Pool, G. J., \& Ruehlman, L. S. (1999). A comparison of the influence of conflictual and supportive social interactions on psychological distress. Journal of Personality, 67(4), 581-622.

Helgeson, V. S., \& Cohen, S. (1996). Social support and adjustment to cancer: Reconciling descriptive, correlational, and intervention research. Health Psychology, 15(2), 135-148.

Ingersoll-Dayton, B., Morgan, D., \& Antonucci, T. (1997). The effects of positive and negative social exchanges on aging adults. Journals of Gerontology: Series B: Psychological Sciences and Social Sciences, 52B(4), S190-S199.

Kelley, H. H., \& Thibaut, J. W. (1978). Interpersonal relationships: A theory of interdependence. New York: Wiley.

Kiecolt-Glaser, J. K., Dyer, C. S., \& Shuttleworth, E. C. (1988). Upsetting social interactions and distress among Alzheimer's disease family care-givers: A replication and extension. American Journal of Community Psychology, 16(6), 825-837.

Krause, N., \& Shaw, B. A. (2002). Negative interaction and changes in functional disability during late life. Journal of Social and Personal Relationships, 19(3), 339-359.

Kuijer, R. G., Ybema, J. F., Buunk, B. P., DeJong, G. M., ThijsBoer, F., \& Sanderman, R. (2000). Active engagement, protective buffering, and overprotection: Three ways of giving support by intimate partners of patients with cancer. Journal of Social and Clinical Psychology, 19, 256-275.

Kurtzke, J. F. (1981). A proposal for a minimal record of disability in multiple sclerosis. Acta Neurologica Scandinavica, 64(87), 110-129.

Lehman, D. R., \& Hemphill, K. J. (1990). Recipients' perceptions of support attempts and attributions for support attempts that fail. Journal of Social and Personal Relationships, 7(4), 563-574.

Lublin, F. D., \& Reingold, S. C. (1996). Defining the clinical course of multiple sclerosis: Results of an international survey. National multiple sclerosis society (USA) advisory committee on clinical trials of new agents in multiple sclerosis. Neurology, 46, 907-911.

Manne, S. L., Taylor, K. L., Dougherty, J., \& Kemeny, N. (1997). Supportive and negative responses in the partner relationship: Their association with psychological adjustment among individuals with cancer. Journal of Behavioral Medicine, 20(2), 101-125.

Murray, T. J. (1995). The psychosocial aspects of multiple sclerosis. Neurology Clinics, 13(1), 197-223.

Newsom, J. T., Nishishiba, M., Morgan, D. L., \& Rook, K. S. (2003). The relative importance of three domains of positive and negative social exchanges: A longitudinal model with comparable measures. Psychology and Aging, 18(4), 746-754.

Pakenham, K. I. (1998). Couple coping and adjustment to multiple sclerosis in care receiver-carer dyads. Family Relations: Interdisciplinary Journal of Applied Family Studies, 47(3), 269-277.

Rasbash, J., Browne, W., Goldstein., H., \& Yang, M. (2000). A user's guide to MlwiN ((2nd ed.)). London: Institute of Education.

Revenson, T. A., Schiaffino, K. M., Majerovitz, D. S., \& Gibofsky, A. (1991). Social support as a double-edged sword: The relation of positive and problematic support to depression among rheumatoid arthritis patients. Social Science \& Medicine, 33(7), 807-813.

Rook, K. S. (1992). Detrimental aspects of social relationships: Taking stock of an emerging literature. In H. O. F. Veiel, \& U. Baumann (Eds.), The meaning and measurement of social support (pp. 157-169). Washington, DC: Hemisphere Publishing Corporation.

Rook, K. S. (2001). Emotional health and positive versus negative social exchanges: A daily diary analysis. Applied Developmental Science, 5(2), 86-97.

Rook, K. S., \& Pietromonaco, P. (1987). Close relationships: Ties that heal or ties that bind. In W. H. Jones, \& D. Perlman 
(Eds.), Advances in personal relationships, vol. 1 (pp. 1-35). Greenwich, CT: JAI Press.

Rook, K. S. (2003). Exposure and reactivity to negative social exchanges: A preliminary investigation using daily diary data. Journals of Gerontology: Series B: Psychological Sciences and Social Sciences, 58B(2), P100-P111.

Rusbult, C. E., \& Van Lange, P. A. M. (2003). Interdependence, interaction and relationships. Annual Review of Psychology, 54, 351-375.

Schuster, T. L., Kessler, R. C., \& Aseltine, R. H. (1990). Supportive interactions, negative interactions, and depressed mood. American Journal of Community Psychology, 18(3), 423-438.

Schwartz, C. E., Foley, F. W., Rao, S. M., Bernardin, L. J., Lee, H., \& Genderson, M. W. (1999). Stress and course of disease in multiple sclerosis. Behavioral Medicine, 25(3), 110-116.

Segrin, C., Badger, T. A., Meek, P., Lopez, A. M., Bonham, E., \& Sieger, A. (2005). Dyadic interdependence on affect and quality-of-life trajectories among women with breast cancer and their partners. Journal of Social and Personal Relationships, 22(5), 673-689.

Shinn, M., Lehmann, S., \& Wong, N. W. (1984). Social interaction and social support. Journal of Social Issues, 40(4), 55-76.

Smith, B. W., \& Zautra, A. J. (2002). The role of personality in exposure and reactivity to interpersonal stress in relation to arthritis disease activity and negative affect in women. Health Psychology, 21(1), 81-88.

Stone, A. A. (1997). Measurement of affective response. In S. Cohen, \& R. C. Kessler (Eds.), Measuring stress: A guide for health and social scientists (pp. 148-171). New York, NY, US: Oxford University Press.

Stone, A. A., Neale, J. M., \& Shiffman, S. (1993). Daily assessments of stress and coping and their association with mood. Annals of Behavioral Medicine, 15(1), 8-16.

Vinokur, A. D., \& Van Ryn, M. (1993). Social support and undermining in close relationships: Their independent effects on the mental health of unemployed persons. Journal of Personality and Social Psychology, 65(2), 350-359.

Watson, D., Clark, L. A., \& Tellegen, A. (1988). Development and validation of brief measures of positive and negative affect: The PANAS scales. Journal of Personality and Social Psychology, 54(6), 1063-1070.

West, S. G., \& Hepworth, J. T. (1991). Statistical issues in the study of temporal data: Daily experiences. Journal of Personality, 59(3), 609-662.

Ybema, J. F., Kuijer, R. G., Hagedoorn, M., \& Buunk, B. P. (2002). Caregiver burnout among intimate partners of patients with a severe illness: An equity perspective. Personal Relationships, 9(1), 73-88. 